\title{
New method of eclipse mapping and an application to HT Cas in the 2017 superoutburst
}

\author{
Yasuyuki Wakamatsu ${ }^{* a}$, Keisuke Isogai $^{a}$, Takashi Morita ${ }^{a}$, Taichi Kato ${ }^{a}$, Daisaku \\ Nogami $^{a}$, Makoto Uemura ${ }^{b}$, Shiro Ikeda ${ }^{c}$ \\ ${ }^{a}$ Kyoto University, Japan \\ ${ }^{b}$ Hiroshima University, Japan \\ ${ }^{c}$ The Institute of Statistical Mathematics, Japan \\ E-mail: wakamatsuekusastro.kyoto-u.ac.jp \\ Isogaidkusastro.kyoto-u.ac.jp, tmoritadkusastro.kyoto-u.ac.jp \\ tkatodkusastro.kyoto-u.ac.jp, nogamidkusastro.kyoto-u.ac.jp, \\ uemuramehiroshima-u.ac.jp, shirodism.ac.jp
}

We have developed a new eclipse mapping method with Total Variation Minimization (TVM). TVM uses a concept of sparse modeling, which recovers information from sparse data. TVM sets a summation of difference in the brightness of adjacent elements in a map to be sparse. We included this concept to the eclipse mapping method and evaluated consistency of the reconstruction of the model disk. The reconstruction of the model light curve seems to be fine but that of the model disk seems to be failed, smearing the brightness distribution along the ingress/egress arcs produced by the shadow of the secondary.

We applied our method to the 2017 superoutburst of HT Cas. The artifacts smearing along the ingress/egress arcs of the secondary also exist. Unaccounted noise and the short phase coverage of the input light curves seem to affect the results, leading to small artificial bright spots in the reconstructed disk.

The Golden Age of Cataclysmic Variables and Related Objects IV

11-16 September, 2017

Palermo, Italy

\footnotetext{
* Speaker.
} 


\section{Introduction}

Accretion disks may play a crucial role in physical phenomena occurring in close binaries. In dwarf novae (DNe), especially, the accretion disk is considered a trigger of its outburst. Thermal instability model suggests that the outbursts in DNe are episodes of mass accretion onto the white dwarf caused by a sudden increase in disk viscosity. The study of accretion disks is, thus, important for an understanding of DNe.

The eclipse mapping method is an indirect image reconstruction technique that reproduces the brightness distribution in cataclysmic variables from the observations of their eclipses [U]. When we observe the binary system at high inclination angle (almost edge-on), the secondary star crosses in front of the accretion disk. We can derive the brightness distribution of the accretion disk occulted by the shadow of the secondary through time-series observations of the eclipse. This is the basic concept of the eclipse mapping method. The information of eclipse is, however, onedimensional data, while that of the brightness distribution in the accretion disk is two-dimensional one, so an additional constraint is required to optimize the distribution map.

A conventional method for this optimization is Maximum Entropy method (MEM). MEM uses an entropy function as an additional constraint and that is defined as

$$
S=-\sum_{j=1}^{N} p_{j} \ln \left(\frac{p_{j}}{q_{j}}\right),
$$

where $N$ is a number of grids in map. $p_{j}$ and $q_{j}$ are defined as

$$
p_{j}=\frac{I_{j}}{\sum_{k} I_{k}}, \quad q_{j}=\frac{D_{j}}{\sum_{k} D_{k}},
$$

respectively. $I_{j}$ is an intensity at $j$-th grid. $D_{j}$ is a default map defined as

$$
D_{j}=\frac{\sum_{k} \omega_{j k} I_{k}}{\sum_{k} \omega_{j k}},
$$

where $\omega_{j k}$ is a weight function in which we include the prior information. Some types of default maps are summarized in [వ].

The consistency between the data and model are measured by a constraint function $\chi^{2}$,

$$
\chi^{2}=\frac{1}{M} \sum_{\phi=1}^{M}\left(\frac{m(\phi)-d(\phi)}{\sigma(\phi)}\right)^{2}=\frac{1}{M} \sum_{\phi=1}^{M} r(\phi)^{2},
$$

where $M$ is a number of data, $m(\phi)$ is a reconstructed model light curve, $d(\phi)$ is a data light curve and $\sigma(\phi)$ is a corresponding uncertainty at the orbital phase $\phi$. It is possible to combine the $\chi^{2}$ with the additional R-statistics, which is defined as

$$
R=\frac{1}{\sqrt{M-1}} \sum_{\phi=1}^{M-1} r(\phi) r(\phi+1) .
$$

These statistics were introduced to minimize the correlation of residuals in the model light curve [B]. MEM chooses the smoothest solution by maximizing the entropy function $S$ under the constraint of the $\chi^{2}$ and $R$. 
The MEM solution is strongly affected by the choice of the weight function and seems less sensitive to small scale brightness structures because the reconstructed map is smoothed and steered towards the default map. There is expected to be, however, local structures in the accretion disk, e.g., a hot spot, which is formed by the collision of the mass transfer stream from the secondary to the disk. Additionally, in SU UMa-type or WZ Sge-type DNe, in which more energetic and long-lasting outbursts called superoutbursts are observed, accretion disks become distorted and the

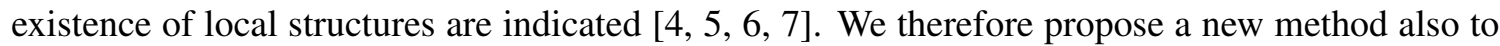
reconstruct these local structures as an alternative to MEM, that is Total Variation Minimization.

\section{Total Variation Minimization}

Total variation minimization (TVM) uses a concept of sparse modeling, which recovers information from sparse data under the assumption of sparseness of information. Here we consider this problem,

$$
\hat{\mathbf{s}}=\underset{\mathbf{s}}{\arg \min }\left(|\mathbf{d}-A \mathbf{s}|^{2}+\lambda_{\mathrm{TV}} \sum_{i, j} \sqrt{\left(s_{i+1, j}-s_{i, j}\right)^{2}+\left(s_{i, j+1}-s_{i, j}\right)^{2}}\right)
$$

where $\mathbf{d}$ is a data light curve having $M$ data set, $\mathbf{s}$ is a brightness distribution having $N \times N$ elements (the map size is $N \times N$ ), and A is an occultation matrix in which the information of shadow of the secondary is included. The first term is an squared term and we minimize this term to derive the solution. However, this is an ill-constrained problem when the number of data $M$ is smaller than the map resolution $N \times N$. This is same as the case of MEM, and thus MEM uses the Entropy function as an additional information for regularization. We now propose the TV term as shown in the second term in equation (2. D) instead of the entropy function. This TV term is a summation of difference in the brightness of adjacent elements. The minimization of this term acts to minimize the difference of adjacent brightness and smooth the whole disk, but it allows the existence of a few large difference at the same time. This behavior is expected to reconstruct the local structure successfully. We regard difference in the brightness of adjacent elements as sparse.

We consider to minimize this cost function composed of the squared and TV term. $\lambda_{\mathrm{TV}}$ is a parameter that sets the strength of the contribution of the TV term, larger $\lambda_{\mathrm{TV}}$ leading to smoother disks.

\section{Results}

We firstly examined the consistency of reconstruction of the model disk. Figure $\mathbb{W}$ shows the reconstruction of disk brightness distribution. Each brightness distribution has two Gaussian sources from which we made model light curves by operating the occultation matrix. We performed the reconstruction for the set of values $\lambda_{\mathrm{TV}}=0.001,0.01,0.1$ and 1 .

We next check the initial condition dependence. The results are showed in Figure $\square$. Other conditions except for initial disk are same as the former case. We set the initial disk as the same one with the model disk.

We finally applied our program to the real data of the 2017 superoutburst in HT Cas. We determined the start and end of each eclipse from the changes in the slope of the light curve. We 

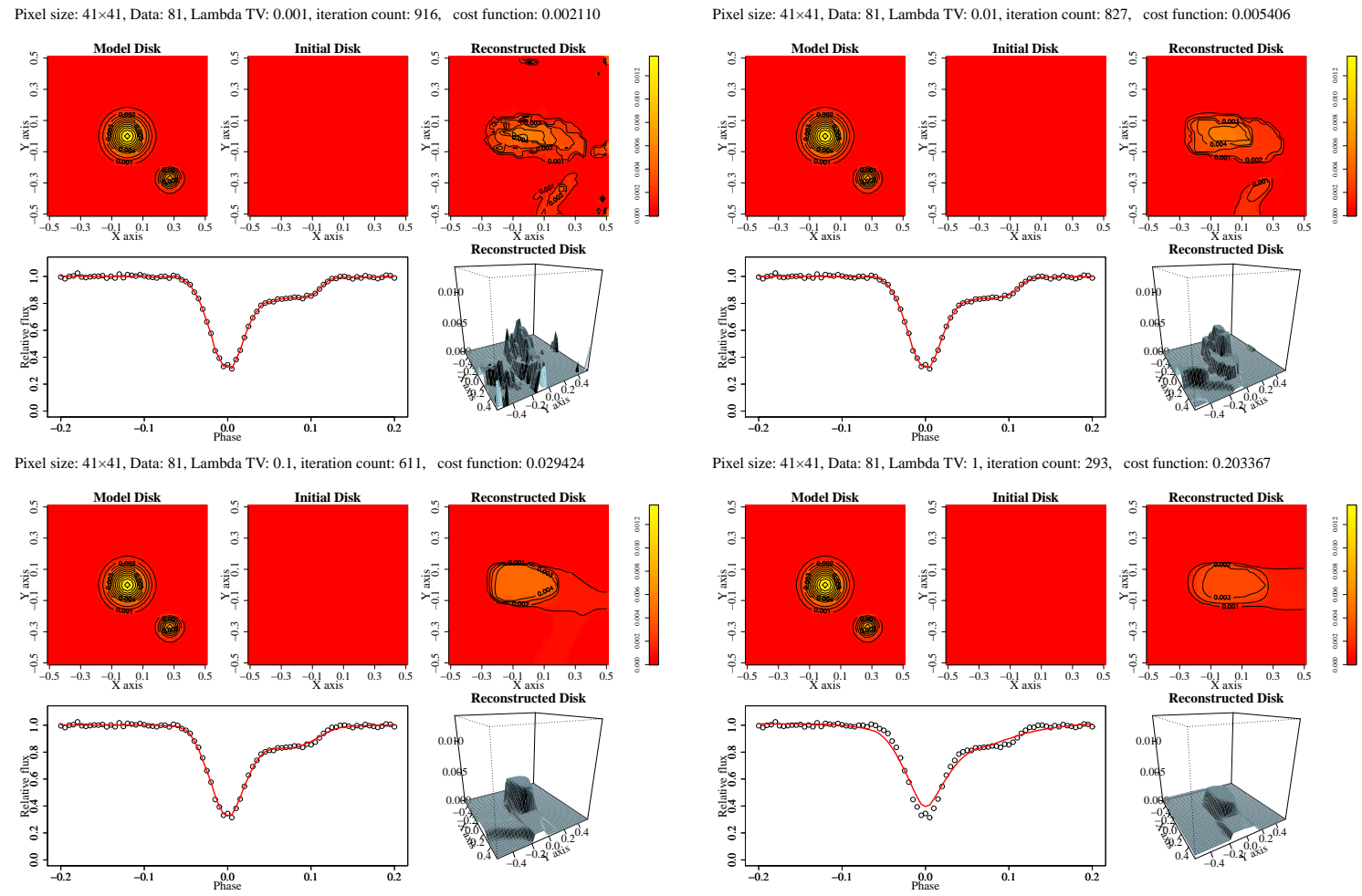

Figure 1: Reconstruction of disk brightness maps from model light curves. The map size is $41 \times 41$ pixels. The model disk has two Gaussian brightness sources (upper-left panels), which was convolved with the occultation matrix to generate an 81 points data light curve. We added an uniform random number as observational error. We estimated the disk brightness distribution (each upper-right and under-right panel) from initial flat maps (each upper-center panel) by optimizing the reconstructed light curve, shown as a red solid line in each light curve. The secondary is to the right of each disk map panel and the binary rotates clockwise.

extracted 4 eclipses through the superoutburst and estimated the brightness distribution in each case. We adopted a fixed $\lambda_{\mathrm{TV}}=0.01$ value and started from the initial map with flat disk. The results are shown in Figure [3.

\section{Discussion}

\subsection{Consistency of TVM}

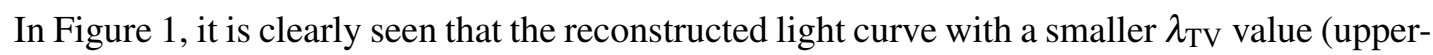
left panel) becomes rougher and it is strongly affected by noise in the light curve, while with a larger $\lambda_{\mathrm{TV}}$ value (lower-right panel) the reconstructed light curve becomes smoother but starts to deviate from the data. The corresponding reconstructed disk also becomes rough in the small $\lambda_{\mathrm{TV}}$ value while it is smooth in the large $\lambda_{\mathrm{TV}}$ value. This trend would reflect the nature of the TV term. These results lead us to choose values in the range $\lambda_{\mathrm{TV}}=0.01-0.1$.

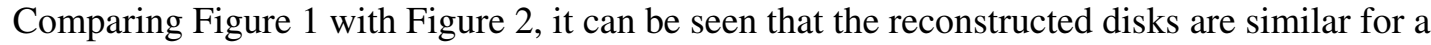
given $\lambda_{\mathrm{TV}}$ value except for the case of $\lambda_{\mathrm{TV}}=0.001$. In this case, the contribution of the TV term is smaller than that in the case of other $\lambda_{\mathrm{TV}}$ values. When the contribution of the TV term is small, 
Pixel size: 41×41, Data: 81, Lambda TV: 0.001, iteration count: 778, cost function: 0.002125
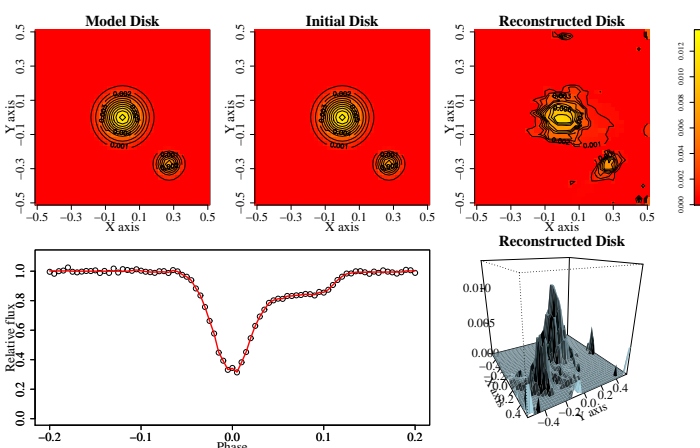

Pixel size: 41×41, Data: 81, Lambda TV: 0.1, iteration count: 790, cost function: 0.029430
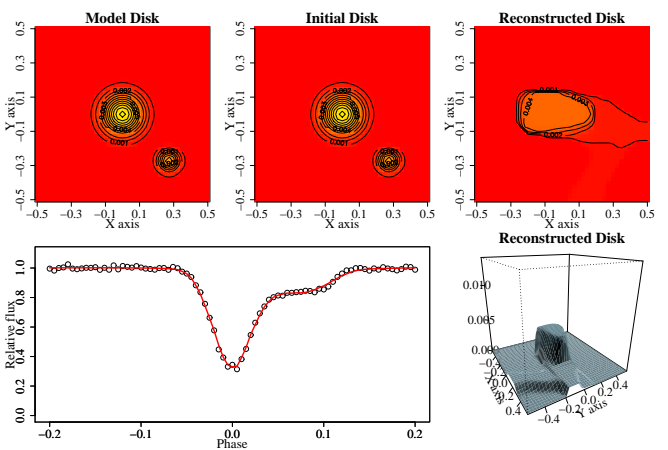

Pixel size: 41×41, Data: 81 , Lambda TV: 0.01 , iteration count: 1285 , cost function: 0.005417
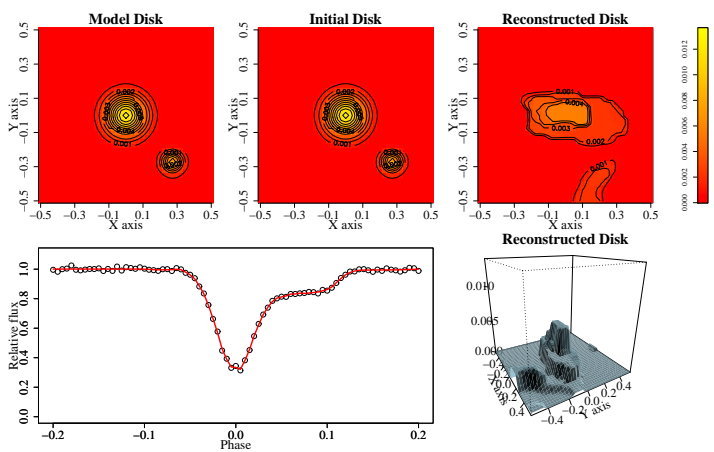

Reconstructed Disk

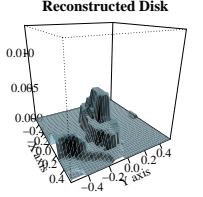

Pixel size: 41 441, Data: 81 , Lambda TV: 1 , iteration count: 442 , cost function: 0.203367
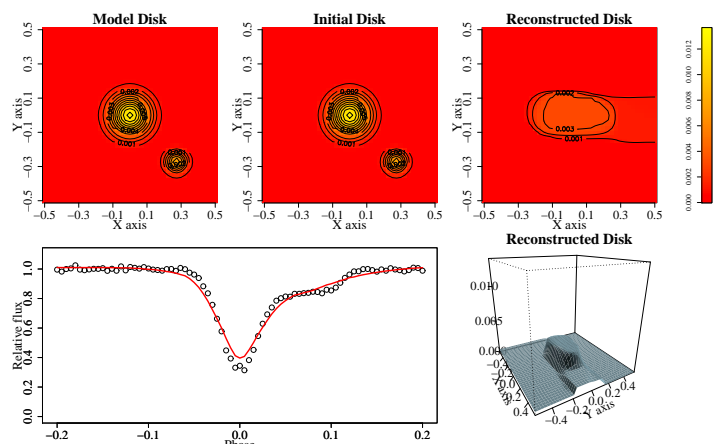

Figure 2: Reconstruction of disk brightness maps from model light curves. We set initial maps as the same maps with model maps and other conditions are same as in Figure $\mathbb{W}$.
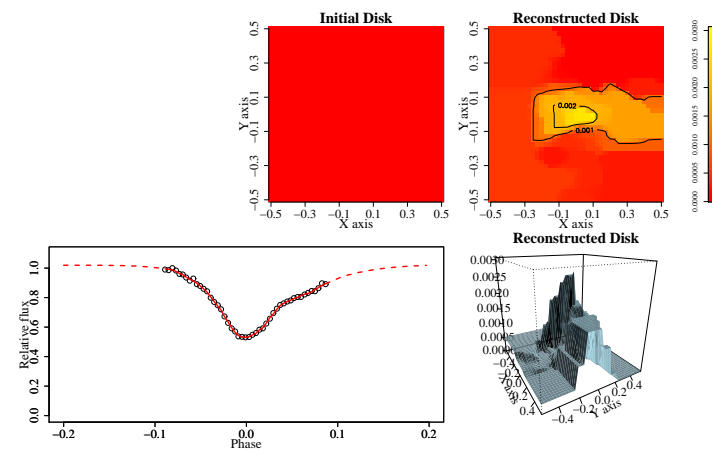

Pixel size: 41×41, Data: 94, Lambda TV: 0.01 , iteration count: 819 , cost function: 0.008884

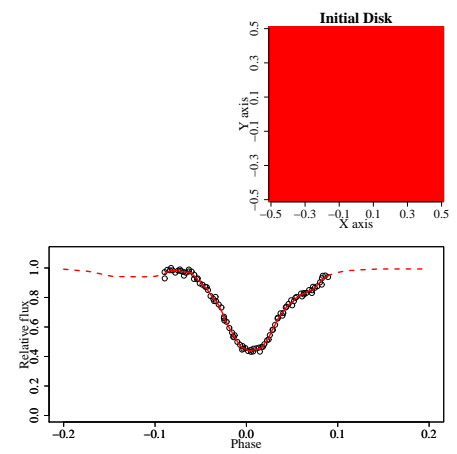

Pixel size: 41×41, Data: 113, Lambda TV: 0.01, iteration count: 853, cost function: 0.007783
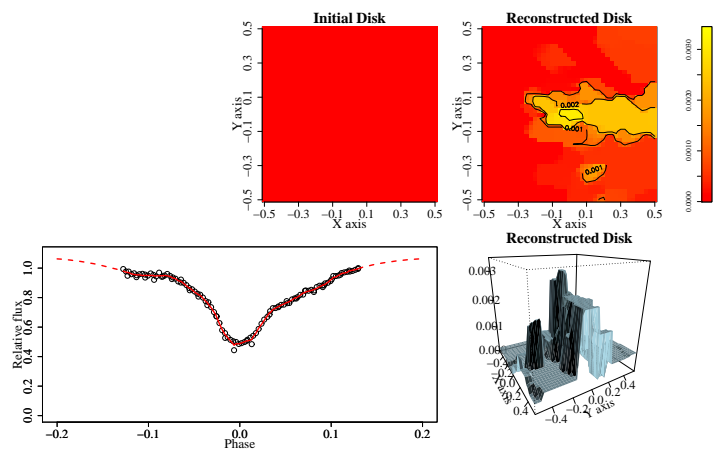

Pixel size: 41 441, Data: 59, Lambda TV: 0.01 , iteration count: 820 , cost function: 0.013925
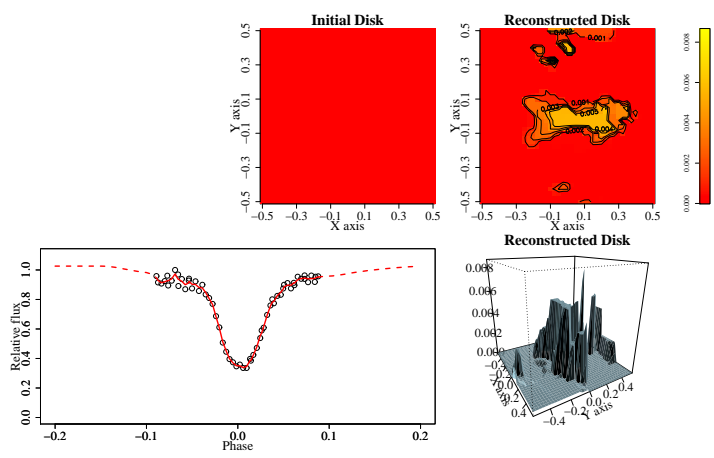

Figure 3: Reconstruction of disk brightness maps from observational light curves. We set initial maps as flat maps and fixed the value of each $\lambda_{\mathrm{TV}}$ in 0.01 . 
the squared term dominates in the cost function and the reconstruction is strongly affected by the initial disk condition. For the remaining $\lambda_{\mathrm{TV}}$ values the reconstructed disks in Figure $\square$ and $\square$ are rather indistinguishable, indicating that the solution is not sensitive to the choice of the initial map

The brightness distribution on the reconstructed disk spreads along the ingress/egress arcs of the shadow of the secondary star. The inconsistency between the model disk and the reconstructed disk may be due to the TV term. Since the TV term prefers a smoother and flatter disk and tends to avoid forming components of large, narrow steep brightness distribution, the resultant disk spreads along the shadow to reduce the peak component, and the TV term makes the brightness components in the disk almost flat. This inherent problem in the TVM eclipse mapping may possibly be alleviated by separating the disk into a main and residual components, and estimating the main one roughly in advance. Since TVM is considered to be incompatible with a power-law disk, it may be effective to reconstruct the main component by assuming the power-law disk, and use TVM method only to estimate the residual component. We finally can derive the whole reconstructed disk by merging these two results. This solution will also be advantageous because the residual component is expected to be small and thus TVM method will work much more effectively.

\subsection{Application to the superoutburst in HT Cas}

The reconstructions of the HT Cas disk seems to be affected by the same brightness spreading along the ingress/egress arcs of the secondary star discussed in Section 4. W. Moreover, the phase coverages are rather small, leading to the lack of information on top and bottom ends in map. We limited the phase coverage of our light curves in order to minimize the influence of superhumps in the reconstruction process. A large phase coverage for eclipse mapping may be obtained by modeling and subtracting the superhump contribution to level the out-of-eclipse brightness of the light curve.

We did not evaluate the noise affecting the data in our eclipse mapping method. The lowerright panel in Figure [3, for example, shows fluctuation in brightness before and after the eclipses, and it affects the reconstruction of light curve and disk, leading to small artificial bright spots in the upper and bottom ends in the map. These components could be removed by evaluating the errors and weighting each data properly.

\section{Summary}

We have developed a new eclipse mapping method with TVM. We evaluated the consistency of reconstruction of the model disk. The reconstruction of the model light curve seems to be fine with proper $\lambda_{\mathrm{TV}}$ values but that of the model disk seems to be failed. Although a structure seemingly like accretion disk can be seen in the center of the map, it spreads along the shadow of the secondary. This is the known effect of criss-crossed arcs which affects eclipse mapping modeling in the absence of an axi-symmetry constraint. The TV term seems to spread the distribution and reduce the peak value probably as the nature of it. This problem may be alleviated by separating the disk component into the main and residual one and estimating the main component roughly by assuming the power-law disk in advance.

We applied our TVM code to the 2017 superoutburst in HT Cas. Besides the spreading of the disk brightness distribution along the ingress/egress arcs of the secondary star, the noise in the data 
light curves and the short phase coverage also affect the reconstruction of the light curve and disk. The effect of noise will be removed by evaluating it properly. The short phase coverage may be alleviated by subtracting the phase-averaged profile of superhumps and leveling the out-of-eclipse flux of the light curves.

\section{References}

[1] Horne, K. 1985, MNRAS, 213, 129

[2] Baptista, R. 2001, Astrotomography, Indirect Imaging Methods in Observational Astronomy, 573, 307

[3] Baptista, R. 1993, A\&A, 277, 331

[4] Whitehurst, R. 1988, MNRAS, 232, 35

[5] Osaki, Y. 1989, PASJ, 41, 1005

[6] Osaki, Y. and Meyer, F. 2002, A\&A, 383, 574

[7] Uemura, M. and Kato, T. and Ohshima, T. and Maehara, H. 2012, PASJ, 64, 92

\section{DISCUSSION}

DMITRY KONONOV: What do you call "true solution"? Is it the global minimum of the regularized functional you use or the solution of the initial problem?

YASUYUKI WAKAMATSU: We consider that the true solution is what has the smallest value of the cost function (Equation (D. $)$ ). The squared term in equation 1 is expected to have very small value if the reconstructed disk same as the model disk are given. The TV term, however, try to choose a smooth disk, probably leading to enlarge the whole value of the cost function. The effect of the TV term is determined by the value of $\lambda_{\mathrm{TV}}$, and it usually determined by the way such as cross validation properly. We finally choose one solution that has the proper value of $\lambda_{\mathrm{TV}}$ and the smallest value of the cost function, and we call it the true solution.

SOLEN BALMAN: Why do you have different size eclipses for HT Cas in your models? During outbursts, eclipses should disappear or diminish largely. During quiescence, eclipses should not really vary. Why are yours varying?

YASUYUKI WAKAMATSU: Light curves during superoutbursts in eclipsing dwarf novae are affected by superhumps, and it is difficult to distinguish eclipses from variations of superhumps. We therefore determined the phase coverage of each eclipse by eye and extracted the parts seemingly eclipses. We wanted to reduce the effect of superhumps on the reconstruction. It is needed to subtract the variation of superhumps from the light curve around the eclipse and level the out-ofeclipse brightness if a larger phase coverage is needed. 